\title{
A Practical Aspect Framework for Enforcing Fine-Grained Access Control in Web Applications
}

\author{
Kung Chen and Chih-Mao Huang \\ Department of Computer Science, National Chengchi University, \\ Wenshan, Taipei 106, Taiwan \\ \{chenk, g9224\}@्cs.nccu.edu.tw
}

\begin{abstract}
Access control is a system-wide concern that has both a generic nature and an application dependent characteristic. It is generic as many functions must be protected with restricted access, yet the rule to grant a request is highly dependent on the application state. Hence it is common to see the code for implementing access control scattered over the system and tangled with the functional code, making the system difficult to maintain. This paper addresses this issue for Web applications by presenting a practical access control framework based on aspect-oriented programming (AOP). Our approach accommodates a wide range of access control requirements of different granularity. AOP supports the modular implementation of access control while still enables the code to get a hold of the application state. Moreover, framework technology offers a balanced view between reuse and customization. As a result, our framework is able to enforce fine-grained access control for Web applications in a highly adaptable manner.
\end{abstract}

\section{Introduction}

The principle difficulty in designing security concern such as access control into an application system is that it is a system-wide concern that permeates through all the different modules of an application. Although there is a generic need to enforce access control for protected resources, yet the specific constraint for granting access to each individual resource may not be the same. Hence in current practices it is very often to see the code for implementing access control scattered over the whole system and tangled with the functional code. This is not only error-prone but also makes it difficult to verify its correctness and perform the needed maintenance; Web applications are no exceptions. Indeed, "broken access control" is listed as the second critical Web application security vulnerability on the OWASP top ten list [15].

A better way to address this problem is to treat security as a separate concern and devise a framework where the generic requirements of access control are captured and the resource-specific constraints can be encapsulated and separated from the functional part of an application [3]. This will not only improve the application's modularity but also make the task of enforcing comprehensive access control more tractable. The Java Authentication and Authorization Services (JAAS) of J2EE [17] is a wellknown attempt toward such a solution. Furthermore, it takes one step forward to pro- 
vide declarative security where access control constraints can be specified declaratively in a configuration file without actual coding. This makes access control highly adaptable.

While sufficient for meeting common access control requirements encountered in Web application development, there are many fine-grained requirements that cannot be satisfied in a modular manner using middleware services such as JAAS. Most notably are the cases when the access control constraints must be defined at the data level. For example, in a B2B E-Commerce site, users from any registered organizations have the privilege to execute the "viewOrder" function, but they are allowed to view only orders of their own organization. This is called instance-level access control constraints [11]. Furthermore, within a data record, certain specific fields, such as credit card number, may have to be excluded from screen view to protect the user's privacy. We refer to this as field-level access control constraints. The declarative security mechanism of JAAS does not cover such fine-grained constraints; to handle them well, we still have to write scattered and tangled code.

It is thus highly desirable to devise a systematic approach and proper mechanisms to realize such fine-grained access control requirements for Web applications in a modular and adaptable manner. We worked toward this goal from two opposite ends and managed to meet in the middle. At one end, the objective is to accommodate requirements. We use a flexible modeling scheme based on user-function-data relationship that can satisfy a wide range of access control requirements of various granularity levels, including both instance and field levels. At the other end, since access control is a system-wide crosscutting concern, we must impose considerable architectural disciplines on Web applications to layout a good foundation for enforcing the required access control modularly. In particular, we follow the well-accepted ModelView-Controller (MVC) [7] architectural pattern and adopt the popular Apache Struts framework [1] to structure our Web applications.

Next, to bridge these two ends, we devise a flexible implementation scheme that does not only support those access control requirements but also integrates seamlessly into the underlying application architecture. Specifically, the emerging techniques of aspect-oriented programming (AOP) [12] are employed to enforce access control constraints in Struts-based Web applications. We take full advantage of AspectJ [13] to design and implement an aspect framework that can meet the diverse information needs of those requirements while allowing reuse and application-specific customization. Furthermore, the codes that implement the required access control are encapsulated and linked to functional modules in a very low-coupling manner, rendering the resulting systems highly modular and adaptable.

The rest of this section gives a brief introduction to AspectJ and the Struts framework. Section 2 describes our approach to access control modeling. Section 3 presents our aspect framework in detail. Section 4 compares related work. Section 5 concludes and sketches future work.

\subsection{AOP and AspectJ}

AOP is a new programming paradigm to support separation of concerns in software development. It addresses the program modularity issues of a crosscutting concern through a new kind of modules, called aspect, and new ways of module composition. 
In AOP, a program consists of many functional modules, e.g. classes in OOP, and some aspects that captures concerns that cross-cuts the functional modules, e.g. security. The complete program is derived by some novel ways of composing functional modules and aspects. This is called weaving in AOP. Weaving results in a program where the functional modules impacted by the concern represented by the aspect are modified accordingly. In languages such as AspectJ, the weaver tool is tightly integrated into the compiler and performs the weaving during compilation.

To facilitate the weaving process, a set of program join points are introduced to specify where an aspect may cross-cut the other functional modules in an application. Typical join points in Aspect J are method execution and field access. A set of join points related by a specific concern are collected into a pointcut. Code units called advice in an aspect are tagged with a pointcut and determine how the application should behave in those crosscutting points. There are three kinds of advice in AspectJ: before, after, and around. The before advice and the after advice are executed before and after the intercepted method, respectively. The case for the around advice is more subtle. Inside the around advice, we can choose to resume the intercepted method by calling the special built-in method proceed(), or simply bypass its execution.

Furthermore, AspectJ also allows aspect inheritance, abstract aspect, and abstract pointcut. We can write an aspect without any reference to a join point by declaring the pointcut abstract. A sub-aspect then extends the abstract aspect and defines the concrete pointcut. As we shall demonstrate later, these abstract aspects open the way to build generic aspects that are essential to an aspect framework.

\subsection{Web Application Architecture and the Struts Framework}

We follow the mainstream approach to Web application architecture that structures an application using the Model-View-Controller (MVC) architectural pattern [7]. The model components encapsulate the application components in the business logic and data tiers. The view components are those pieces of an application that display the information provided by model components and accept input. The controller is a special program unit that coordinates all the activities of the model and view components. It acts as a central point of control within an application.

Currently, most Web application frameworks are MVC-based. In particular, we choose the popular open source Struts framework [1]. It is compatible with Sun's J2EE platform and primarily based on Servlet [19] and JSP [18] technology. Figure 1 illustrates the structure of a Struts-based Web application. The controller is implemented using a dedicated ActionServlet with the assistance of an XML configuration file, struts-config.xml. Every user request is dispatched to an Action class by the ActionServlet according to the action mapping defined in the configuration file. These actions are responsible for serving user requests or passing them to the correct business model components, and for returning the correct view element that the ActionServlet should forward to afterwards. This view forwarding is also based on the mapping information specified in the configuration file. The view element is often a JSP page, extended with custom tag libraries provided by Struts. 


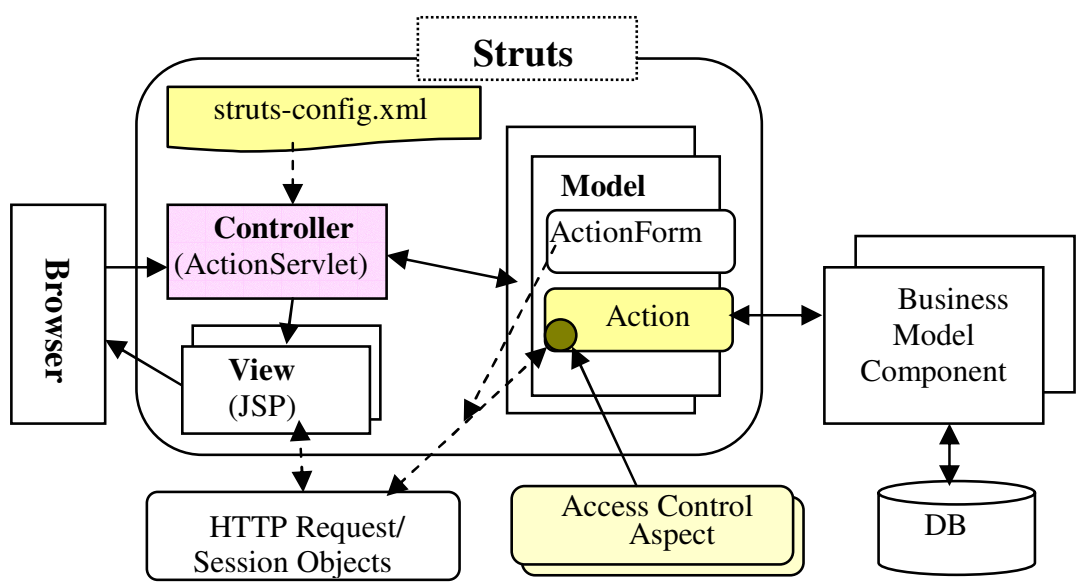

Fig. 1. Struts-Based Web application architecture

Moreover, the HTTP request object and the session object provided by the Servlet framework serve as the shared data repository between action classes and JSP views. User input captured via an ActionForm is stored in the two objects; action classes also put their execution results in these objects. Hence JSP views can fetch both user input and execution results from them for display. For example, user authentication results and user profiles are kept in the session object for authorization and other purposes.

\section{Access Control Modeling}

Access control, also known as authorization, is a process by which users, after being identified, are granted certain privileges to information, system functions, or resources. The step to identify a user is usually called authentication. Username and Password check is the most basic form of authentication, while digital certificates and biometrics are more advanced ways of authentication.

Since RBAC [16], there have been many approaches proposed to model access control requirements for applications purposes. Here we take a simple yet generic approach that can support a wide range of access control requirements. We model the interaction between a user and a Web application as a sequence of access tuples of three elements: <user, function, data>, indicating a user's request to execute the function on a specific type of data object(s). The access control rules of an application determine which access tuples are allowed and which must be denied. They are derived from the application's access control requirements.

In designing the form of our access control rules, we focus on the functionalities of an application and specify the access control rules in a function-oriented manner. Furthermore, as authentication is required prior to authorization, we also make " $\mathrm{Au}$ thenticationType" part of the rule; the type can be id/password (PWD), digital certificate (DC), or any other supported methods of user identification. Specifically, the access control rules take the following form:

Rule: <funName, authType, dataClassName[-fieldNames], constraint > 
Here funName is the name of a function whose access needs to be restricted, authType is the required user authentication type, and dataClassName refers to the type of the data objects being operated by funName. Note that [-fieldNames] following dataClassName is optional; when present, it specifies the list of fields whose contents must be masked before presenting the data object(s) in context to the user. Last, the constraint is a Boolean expression which must be evaluated to true to grant the attempted access. We assume a few basic relational operators, such as equal, less and set membership, available for specifying the constraint expression. If the constraint does not refer to any information on the data object, the dataClassName element can also be left unspecified as "_,".

Clearly, the more related entities we can refer to in the constraint expression the wider the scope of access control requirements we can support. For generic purposes, we take an object-based approach to specify the constraints and supply three generic objects: User, Fun, Data, with various attributes that the constraint expression can refer to. Typical attributes for the User object include user's name and roles in an organization. The attributes of the Fun object include the function's full name and the arguments passed to it, while the fields of the dataClassName are the default attributes of the Data object to support fine-grained control. The specific set of attributes depends on individual application's needs.

Furthermore, to accommodate more access control requirements, we provide another two objects for specifying the constraints. First, the context object $(C x t)$ provides methods to retrieve the time and location of the attempted access. This is the most often used contextual information for access control. Second, the application object $(A p p)$ is global to an application and stores various specific parameters related to access control. For example, certain functions are accessible only during working days and from specific machines. These application-wide parameter definitions can be provided easily through a standard Java property file.

Example: The following is a set of access control requirements and corresponding rules for a typical online shopping and order management system. Note that " $\& \&$ " stands for the and operator, and "ll" the or operator.

C1: Only registered (authenticated) users can search the detailed product catalog.

R1: <searchDetailedCatalog, PWD, , true >

C2: All registered users (a.k.a. customer) can create order, but only VIP customers can create orders whose total amount exceed $\$ 100,000$.

R2: <createOrder, PWD, _, ( lessEq(Fun.getArgument(“total”), 100000) Il equals(User.getAttr( "VIP”), true) ) >

C3: Only sales managers authenticated through digital certificates can delete order objects.

R3: <deleteOrder, DC, Order, contains(User.getAttr( "Roles”), “Sales”) \&\& contains(User.getAttr("Roles”), “Manager”)>

C4: Customers can list (view) their own orders, but the credit card number should be excluded from display. 
R4: <listOrders, PWD, Order[-creditCardNumber], equals(User.getAttr("Name”), Data.getAttr("Owner"))>

C5: Unclassified orders can be printed in batch mode by sales from dedicated machines during working days.

R5: < batchPrintOrder, PWD, Order, contains(User.getAttr("Roles"), "Sales")

\&\& contains(App.getAttr("WorkingDays"), Cxt.getDay())

$\& \&$ contains(App.getDedicatedMachines(), User.getAttr( “clientIP”))

$\& \&$ equals(Data.getAttr( "SecurityLevel"), "Unclassified")>

This form of access control rules is very flexible and can model a multitude of requirements, from simple RBAC to sophisticated instance and field level constraints.

\section{Building the Aspect Framework}

This section describes our design of the aspect framework and presents it in detail.

\subsection{Choosing the Pointcuts}

As stated earlier, access control is a cross-cutting concern that AOP aims to modularize well. Yet, it is not something like function tracing that is completely orthogonal to the functions and all one has to do in AOP is to define the functions to be traced as the pointcuts without any involvement form the underlying functions. In contrast, access control decisions, especially fine-grained ones, need substantial information from the application about the context in which the decisions are required. Hence access control aspects depend on the cooperation from the application to a significant degree. On the other hand, as we are not inventing a totally new style of application architecture, we need to carefully look for the proper pointcuts to weave in aspects so that a suitable balance between information need and non-invasiveness is achieved.

Given the requirements above, central to our design of the aspect framework is to determine which type of program units in a Struts-based application to weave in the aspect code. This in turn is greatly influenced by the following design considerations. First of all, all the information required for enforcing access control rules and filtering out unauthorized contents must be available to the aspect code, since fine-grained access control cannot be realized without detailed application state. Second, in case that the attempted access must be denied, exception propagation and handling must not incur significant impacts on the program structure. Third, the correspondence between an access control rule and an enforcing aspect should be direct and clear for management and maintenance purposes.

Based on these considerations, we conducted some experiments and investigation. Finally, we choose the user action classes in Struts as the targets for access control aspect weaving. Action classes play the role of gateway between the presentation tier 
and the business and the data tiers. All action classes must inherit from the class Action and implement an execute method ${ }^{1}$ with the following signature:

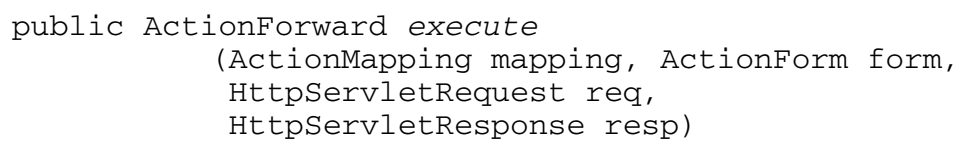

It turns out that the execute method is indeed the proper join point to weave in our advice code for its arguments expose the right information we need. Firstly, user input and any intermediate results, including user authentication records and user profiles, are all stored in the request or session objects, which are available through the HttpservletRequest argument, req. Therefore, via the args () pointcut of AspectJ, our aspects will have all the information needed for enforcing the access control and filtering out unauthorized contents.

Secondly, the control transfer between actions and views is specified in the strutsconfig.xml file, and is realized through the action mapping argument passed to the method and the Actionforward object returned by an action class. Hence we can define proper exception pages in the configuration file and forward to them when an access request must be denied. Apparently, this exception handling scheme fits very well into Struts. Lastly, as will be shown later, an access control rule corresponds to its enforcement aspect quite directly since a user function will usually be supported by an action class.

\subsection{Constructing the Aspects}

Having chosen the user actions as our weaving targets, the next step is to investigate the structure of access control aspects for the framework's purpose. Following the general spirit of framework construction [4][6], we shall divide the aspect code into two parts: generic part realized by abstract aspects and rule specific part realized by concrete aspects. This division is derived from a detailed analysis of the nature of information needed for enforcing the various kinds of access control rules presented in Section 2. The rule specific part of an aspect is easier to grasp. It is obvious that the authentication type, pointcut definitions, the constraint to check, and the removal of unauthorized contents are specific to each individual rule. The interesting case is the generic part of the access control aspects. For this, we must look into the inner structure of the access control rules and constraints.

\subsubsection{Authentication Aspects}

Firstly, the simplest type of access control is indeed user authentication. Here the main concern is how to accommodate different schemes of user authentication. Besides, since all access controls must be preceded by proper user authentication, we shall make the various authentication codes available to all aspects in the framework. This is achieved by defining a root aspect that also acts as the factory of authentication objects. We call this root aspect AAAspect ${ }^{2}$ and here is its code:

${ }^{1}$ Since Version 1.1, Struts also supports method-based dispatch units through a new class called DispatchAction. This can also be covered by our framework with little adaptation.

${ }^{2}$ For spaces' sake, we omit the four arguments captured at the pointcuts (the execute() method). 


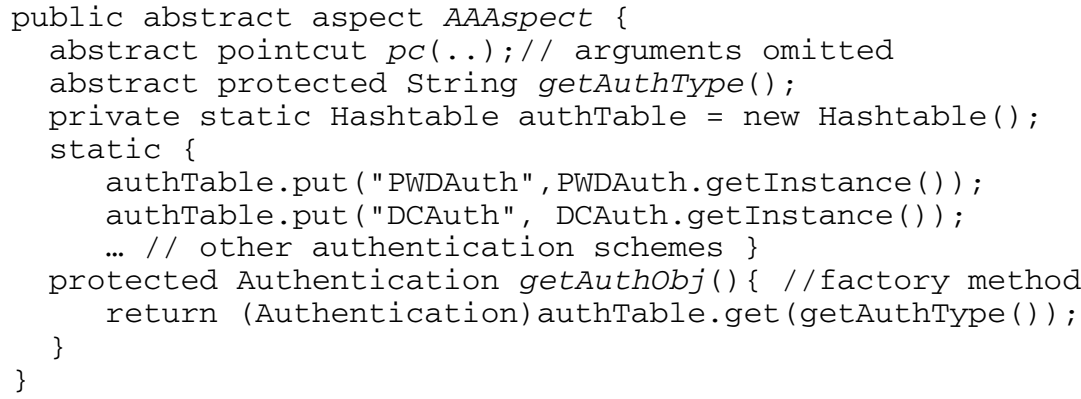

The getAuthobj ( ) method is the factory method that will return the right authentication object according to the result of executing the getAuthType () method, which is rule specific and defined in concrete aspects. For each authentication type, there will be a singleton authentication object containing the code to verify that a user has passed the identification check and the login page name for re-direction purpose in case the user has not. These objects are instantiated from subclasses of the Authentication class, which prescribes the interface for verifying authentication checks (i.e., isAuthenticated()) and for obtaining the re-direction page (i.e., forwardTo ()). In the code listing above, we have included two such objects instantiated from class PWDAuth and class DCAuth.

The user authentication task is a coordinated work between authentication aspects and authentication objects. Authentication aspects are responsible for invoking the methods of right authentication objects to perform the check at right places according to the access control rules. We factor out the common code of authentication aspects into the Authentication aspect and leave to its sub-aspects, e.g., PWDAuthAspect, the specifications regarding where to do the checking (pointcut) and which type of authentication to use. Here is the code for the Authentication aspect:

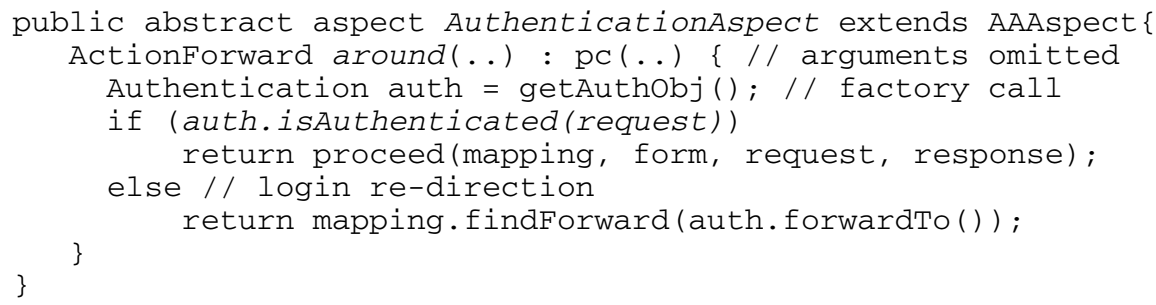

\subsubsection{Access Control Aspects}

The structure of our access control aspects is determined by the constraints in access control rules. Specifically, the availability of the information referenced in the constraints, such as user roles, function arguments and data contents, will shape the structure of our aspect codes. We now explain how this relates to our design as follows.

As described earlier, in Struts-based Web applications, user profile information, user input, and any execution results are all kept in the session object or the request object, hence accessible to the aspect $\operatorname{code}^{3}$. The real issue here is when the required

\footnotetext{
${ }^{3}$ The Cxt and App objects are of global type, so they are always available.
} 
information is available. Previous work [4][5] tacitly assumed that the information is available to the aspect code for making the access control decision before executing the designated function. However, this is not the case for content-based fine-grained access control requirements. For instances, both rule R4 and rule R5 given in Section 2 need to examine the data contents to filter out unauthorized data. Such constraints cannot be enforced without first invoking the function requested.

A close look at the constraint expressions of R4 and R5 reveals that it is the reference to the attributes of the Data object that calls for additional data filtering. Indeed, unlike the attributes of the User and Fun objects, the attributes of the Data object are not available before executing the designated function. Therefore, we propose two different kinds of access control aspects for realizing these requirements, namely prechecking and post-filtering. The pre-checking aspects will handle the common cases where the constraint involves only the User and Fun objects while the post-filtering aspects are needed if the constraint also refers to the attributes of the Data object.

Listing 1. The PostfilteringCollection aspect

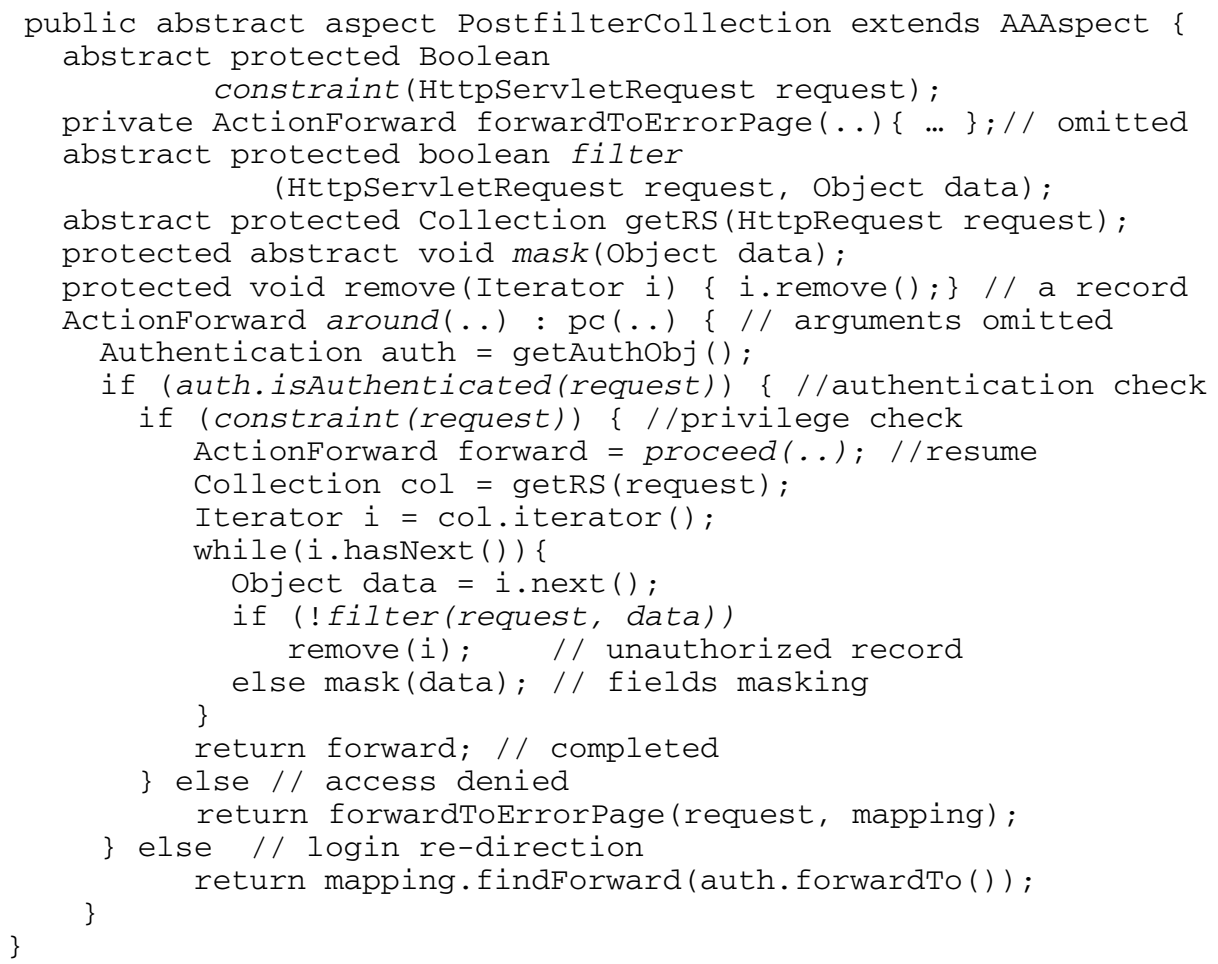

Both pre-checking and post-filtering aspects must first get an authentication object and make a call to its isAuthenticated() method. This will ensure that access control enforcement is preceded by user authentication. Moreover, the advice in the post-filtering aspects must also conduct an access constraint check before proceeding 
to execute the designated function and filter out unauthorized contents ${ }^{4}$. Since prechecking aspects is the base for post-filtering aspects, we shall only present postfiltering aspects due to the space limitation.

As the code structure for handling a single record retrieved by key-based queries is different from that of handling a collection of data obtained by ad-hoc queries, we further divide the post-filtering aspect into two styles: single and collection. Listing 1 shows the code of the PostfilterCollection aspect. Inside the advice, after performing the authentication and access checks, it resumes executing the intercepted function, retrieves the query results from the request object and iteratively applies the filter and the field mask to each individual data record. The specific definitions of the constraint expression, filter condition and fields mask are left to concrete aspects that inherit it. The code for PostfilterSingle is very similar yet simpler, since only one data record is being examined.

Figure 2 shows the whole aspect framework and depicts how it works. In summary, to realize a particular access control rule, one has to define a concrete aspect that inherits from a proper abstract aspect, and provide the definitions of pointcut, constraint method, filter condition, and field masking method, if needed.

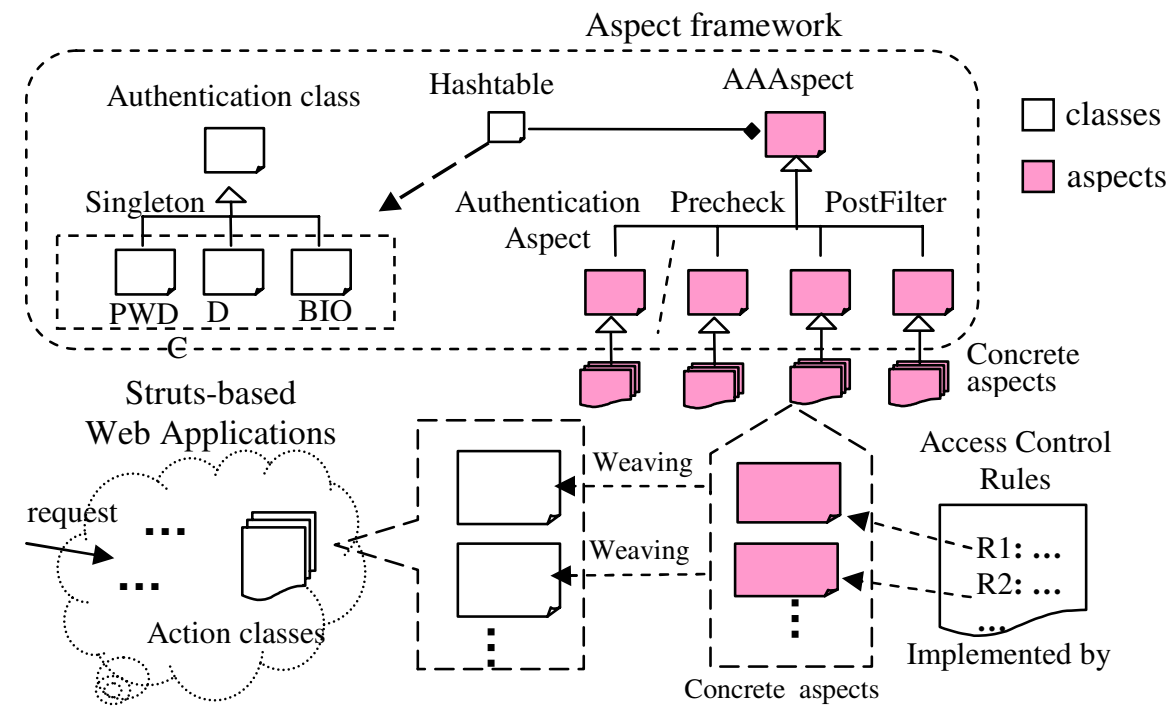

Fig. 2. The structure and operation of the aspect framework

\section{Related Work}

Role-based access control (RBAC) [16] is the most often cited guiding principle for application-level security. Since then there are many extended proposals for modeling

\footnotetext{
${ }^{4}$ This is indeed undesirable code duplication caused by the lack of proper advice reuse mechanisms is AspectJ. But this issue is beyond the scope of this paper.
} 
fine-grained access control. We have role-templates [10], domain-type enforcement [2], content-based [20], team-based [9], and instance-level [11], just to name a few. Basically, they all attempt to base access control constraints on some more detailed relationships among the user, the function requested, and the data to be accessed, as we did here. Context information for access control is investigated in [9] [14] [20].

Our work bears a closer relationship with that of Goodwin et al [11]. First, they used the four-tuple access control rules: [user group, action, resource, relationship], where a set of predefined relationship, as opposed to our general constraint, is defined for each resource type. The major concern is instance-level constraints, no field-level constraint covered, though. Second, they also adopt an MVC-like architecture. The controller intercepts all user commands and queries a centralized manager to make the authorization decision. But they did not consider different authentication types and neither did they use aspect-oriented programming to build their framework.

Applying AOP to security concerns is pioneered by [4] [5]. They also sketched how to build frameworks in AspectJ for handling access control. However, they did not focus on Web applications, and neither did they look into access control modeling in detail as we did. The proposed aspects check the constraint before the attempted access and use per-object stateful aspects. In contrast, we have both pre-checking and post-filtering aspects and use stateless singleton aspects. Hanenberg et al [6] describes some idioms for building framework in AspectJ. Georg et al. [8] studies the use of aspects for modeling security concerns from system analysis perspective.

\section{Conclusion and Future Work}

Security is attracting more and more concerns in the development of Web applications. However, current practices are not capable of supporting a modular implementation of fine-grained access control for Web applications. In this paper, we have presented a systematic approach to model fine-grained access control requirements and an aspect framework for enforcing such requirements for Struts-based Web applications. Our modeling scheme can satisfy a wide range of requirements with different granularity. By employing AOP and framework technology, we have obtained a highly modular implementation of fine-grained access control and achieved a good balance between reuse and customization. Furthermore, the correspondence between access control rules and associated aspects is direct and hence easy to adapt.

A direction we plan to explore is to prepare the access control rules in a configuration file and develop a declarative implementation scheme. Currently, the access control aspects are manually derived from the rules, which require certain programmatic efforts. We shall investigate a translation scheme to generate the concrete aspects automatically from access control rules. This will lead us toward a more declarative implementation and greatly improve the manageability and maintainability of access control requirements.

Acknowledgements. This work was supported in part by the National Science Council, Taiwan, R.O.C. under grant number NSC-93-2213-E-004-009. 


\section{References}

[1] The Apache Struts Web Application Framework: http://struts.apache.org/

[2] Chandramouli, R.: A Framework for Multiple Authorization Types in a Healthcare Application System, 17th Annual Computer Security Applications Conference, Dec. 2001.

[3] De Win, B., Piessens, F., Joosen, W. and Verhanneman, T., On the importance of the separation-of-concerns principle in secure software engineering, Workshop on the Application of Engineering Principles to System Security Design, 2002.

[4] De Win, B., Vanhaute, B., and De Decker, B., Building Frameworks in AspectJ, ECOOP 2001, Workshop on Advanced Separation of Concerns, pp.1-6.

[5] De Win, B., Vanhaute, B. and De Decker, B., Security Through Aspect-Oriented Programming, Advances in Network and Distributed Systems Security, Kluwer Academic, pp. 125-138, 2001.

[6] Hanenberg, S. and Schmidmeier, A., Idioms for Building Software Frameworks in AspectJ, 2nd AOSD Workshop on Aspects, Components, and Patterns for Infrastructure Software (ACP4IS), Boston, MA, March 17, 2003.

[7] Gamma, Helm, Johnson and Vlissides, Design Patterns. Addison-Wesley, 1995.

[8] Georg, G., Ray, I., and France, R., Using Aspects to Design a Secure System, Proc. of the 8th IEEE Int'l Conf. on Engineering of Complex Computer Systems. December 2002.

[9] Georgiadis, C.K., Mavridis, I., Pangalos, G., and Thomas, R.K.: Flexible Team-based Access Control Using Contexts, Sixth ACM Symposium on Access Control Models and Technologies (SACMAT 2001), Chantilly, VA, USA, May 2001.

[10] Giuri, L., and Iglio, P., Role Templates for Content-Based Access Control, Proceedings, 2nd ACM Workshop on Role-Based Access Control, Fairfax, VA (October 28-29, 1997), pp. 153-59.

[11] Goodwin, R., Goh, S.F., and Wu, F.Y., Instance-level access control for business-tobusiness electronic commerce, IBM System Journal, vol. 41, no. 2, 2002.

[12] Kiczales, G., Lamping, J., Menhdhekar, A., Maeda, C., Lopes, C., Loingtier, J.-M., and Irwin, J., Aspect-Oriented Programming, in ECOOP '97, LNCS 1241, pp. 220-242.

[13] Kiczales, G., Hilsdale, E., Hugunin, J., Kersten, M., Palm, J., and Griswold, W.G., Getting Started with AspectJ, Communications of ACM, vol. 44, no. 10, pp 59-65, Oct. 2001.

[14] Kouadri Mostefaoui, G., and Brezillon, P., A generic framework for context-based distributed authorizations, In: Modeling and Using Context (CONTEXT-03), LNAI 2680, Springer Verlag, pp. 204-217.

[15] Open Web Application Security Project: The Top Ten Most Critical Web Application Security Vulnerabilities. http://www.owasp.org/documentation/topten

[16] Sandhu, R., Coyne, E., Feinstein, H., and Youman, C., Role-Based Access Control Models, IEEE Computer, 29(2):38-47, 1996.

[17] Sun Microsystems, Java Authentication and Authorization Service (JAAS), http://java.sun.com/products/jaas/index.jsp

[18] Sun Microsystems, JavaServer Pages Technology (JSP): http://java.sun.com/ products/jsp/

[19] Sun Microsystems, Java Servlet Technology : http://java.sun.com/products/servlet/

[20] Tzelepi1, S.K., Koukopoulos, D.K., and Pangalos, G.: A flexible Content and Contextbased Access Control Model for Multimedia Medical Image Database Systems. ACM SIGMM Electronic Proceedings, 2001. 